Algebraic 83 Geometric $\mathcal{T}$ opology

Volume 5 (2005) 1291-1314

Published: 6 October 2005

ATG

\title{
Almost integral TQFTs from simple Lie algebras
}

\author{
QI CHEN \\ Thang Le
}

\begin{abstract}
Almost integral TQFTs were introduced by Gilmer [G]. The aim of this paper is to modify the TQFT of the category of extended 3cobordisms given by $[\mathrm{T}$ to obtain almost integral TQFT.
\end{abstract}

AMS Classification 57M27, 57R56

Keywords TQFT, almost integral TQFT, simple Lie algebra

\section{Introduction}

Inspired by a 3-dimensional interpretation of the Jones polynomial for knots, Witten predicted that one can define topological invariants for 3-manifolds using simple complex Lie algebras. The first concrete construction was obtained by Reshetikhin and Turaev [RT] for $s l_{2}$. Soon after, similar invariants were constructed for all simple Lie algebras. They are called the Witten-ReshetikhinTuraev invariants (WTR-invariants for short) or quantum invariants because Reshetikhin and Turaev's construction is based on the theory of quantum groups. It was also Witten's vision that the WTR-invariants can be extended to the Topological Quantum Field Theory (TQFT) in a rather natural manner. Loosely speaking, a TQFT with base ring $K$ is a functor from the category $\mathcal{C}$ to the category of $K$-modules where the objects in $\mathcal{C}$ are surfaces and morphisms in $\mathcal{C}$ are 3 -dimensional cobordisms. We denote a TQFT by a pair $(\mathcal{T}, \tau)$ where $\mathcal{T}$ and $\tau$ are maps between objects and morphisms respectively. Most known TQFTs have anomalies. Anomalies can be resolved by introducing $p_{1}$-structure as in BHMV or by studying the category of extended 3-cobordisms as in [T]. In this paper we follow the latter. One of the features of extended 3-cobordisms is that they contain embedded colored ribbon graphs. (See Section 2.2 for more details about TQFT and ribbon graph.) For each simple complex Lie algebra $\mathfrak{g}$ and certain integer $r$, one can define a TQFT $\left(\mathcal{T}_{r}^{\mathfrak{g}}, \tau_{r}^{\mathfrak{g}}\right)$. In this case, the colors of the ribbon graphs come from the representations of the quantum group $\mathbf{U}_{v}(\mathfrak{g})$ associated to $\mathfrak{g}$ with the parameter $v$ equal to a primitive $r$-th root of unity. 
Gilmer introduced the notion of almost integral TQFT in $\mathrm{G}$. Let $D$ be a Dedekind domain contained in a ring $K$. A TQFT $(\mathcal{T}, \tau)$ with base ring $K$ is called almost ( $D$-)integral if there exists some element $\mathcal{E} \in K$ such that $\mathcal{E} \tau(M)$ is in $D$ for any closed connected cobordism $M$. (Note that in $\mathrm{G}$, $\mathcal{E}$ is required to be in $D$. One can show that most results in [G] hold in this more general definition.) Gilmer showed that the TQFTs considered in [BHMV] are almost integral. Using this, Gilmer, Kania-Bartoszynska and Przytycki GKP proved that for a prime $p>2$ if an integral homology 3 -sphere is $p$-periodic, i.e. it admits a $\mathbb{Z} / p \mathbb{Z}$ action with fixed point set a circle, then its projective $s l_{2}$ (or $\mathrm{SO}_{3}$ ) WRT-invariant satisfies some congruent relation. This criterion is used to show that the Poincare sphere is 2,3 , and 5 -periodic only in $\mathrm{CL}$.

In this paper, we show, in Theorem 3.2, that a modified version of the TQFT $\left(\mathcal{T}_{r}^{\mathfrak{g}}, \tau_{r}^{\mathfrak{g}}\right)$ is almost integral. The modification is to restrict the colors for ribbon graphs embedded in extended 3-cobordisms. The difficulty in proving Theorem 3.2 is that one must show the integrality of the projective WRT-invariant for every closed 3-cobordism with a ribbon graph, while it was sufficient in [G] to show the integrality for every closed 3-cobordism with a framed link. (Framed links are special ribbon graphs.) Theorem 3.2 is an important ingredient to prove that certain TQFTs with rings of algebraic integers as base rings can be constructed from $\left(\mathcal{T}_{r}^{\mathfrak{g}}, \tau_{r}^{\mathfrak{g}}\right)$, cf. [C]. As a corollary of Theorem 3.2 the criterion in GKP can be generalized to all simple Lie algebras (Corollary 4.1).

We start by recalling necessary definitions in Section 2 We state the main results in Section 3. We will discuss one application of Theorem 3.2 in Section 4 . Section 5 contains the proof of Theorem 3.2

The authors wish to thank the referee for numerous helpful comments.

\section{Preliminaries}

In this section, we recall some definitions and known results needed in this paper.

\subsection{Lie algebras and their quantum deformations}

We will define quantum group and then look at its representation theory. The reader may skip this section if he or she is familiar with it.

Let $\mathfrak{g}$ be a simple complex Lie algebra with Cartan matrix $\left(a_{i j}\right), i, j=1, \ldots, \ell$. Fix a Cartan subalgebra $\mathfrak{h}$ of $\mathfrak{g}$ and a set of simple roots $\Pi_{\mathfrak{h}}=\left\{\alpha_{1}, \ldots, \alpha_{\ell}\right\}$ 
in its dual space $\mathfrak{h}^{*}$. One can define a symmetric bilinear form $(\cdot \mid \cdot)$ on $\mathfrak{h}^{*}$ in the following way. Multiply the $i$-th row of $\left(a_{i j}\right)$ by $d_{i} \in\{1,2,3\}$ such that $\left(d_{i} a_{i j}\right)$ is a symmetric matrix. Set $\left(\alpha_{i} \mid \alpha_{j}\right)=d_{i} a_{i j}$. This bilinear form is proportional to the dual of the Killing form restricted on $\mathfrak{h}$. Let $X$ and $Y$ be the weight lattice and the root lattice of $\mathfrak{g}$. The Weyl group $W$ acts on $X$ and $Y$ naturally. The order of the group $X / Y$ is $\operatorname{det}\left(a_{i j}\right)$. Let $X_{+}$be the set of dominant weights and $Y_{+}=Y \cap X_{+}$. According to the general theory of Lie algebra, the finite-dimensional representations of $\mathfrak{g}$ are parameterized by the dominant weights. Let $d=\max _{1 \leq i \leq l}\left\{d_{i}\right\}$. Then $d$ is the square of the ratio of the lengths of a long root and of a short root of $\mathfrak{g}$. Let $\alpha_{0}$ be the highest short root, and let $\rho$ be half of the sum of positive roots. The dual Coxeter number is $h^{\vee}=1+\max _{\alpha>0}(\alpha \mid \rho) / d$.

From now on we fix a simple Lie algebra $\mathfrak{g}$. Let $h$ be a formal parameter and $\mathbf{U}_{h}=\mathbf{U}_{h}(\mathfrak{g})$ be the $\mathbb{C}[[h]]$-algebra topologically generated by the set of generators $\left\{X_{i}, Y_{i}, H_{i}\right\}_{1 \leq i \leq l}$ and the relations

$$
\begin{array}{ll}
{\left[H_{i}, H_{j}\right]=0,} & {\left[X_{i}, Y_{j}\right]=\delta_{i j} \frac{\sinh \left(h d_{i} H_{i} / 2\right)}{\sinh \left(h d_{i} / 2\right)},} \\
{\left[H_{i}, X_{j}\right]=a_{i j} X_{j},} & {\left[H_{i}, Y_{j}\right]=-a_{i j} Y_{j}}
\end{array}
$$

and if $i \neq j$

$$
\begin{aligned}
& \sum_{k=0}^{1-a_{i j}}(-1)^{k}\left[\begin{array}{c}
1-a_{i j} \\
k
\end{array}\right]_{v_{i}} X_{i}^{k} X_{j} X_{i}^{1-a_{i j}-k}=0 \\
& \sum_{k=0}^{1-a_{i j}}(-1)^{k}\left[\begin{array}{c}
1-a_{i j} \\
k
\end{array}\right]_{v_{i}} Y_{i}^{k} Y_{j} Y_{i}^{1-a_{i j}-k}=0
\end{aligned}
$$

where $v_{i}=e^{h d_{i} / 2}$ and $\left[\begin{array}{l}a \\ b\end{array}\right]_{x}=[a]_{x}^{!} /[b]_{x}^{!}[a-b]_{x}^{!}$for $a \geq b \geq 0$. Here $[y]_{x}^{!}=$ $\prod_{i=1}^{y}[i]_{x}$ and $[z]_{x}=\left(x^{z}-x^{-z}\right) /\left(x-x^{-1}\right)$. We use $[a]_{i}$ to denote $[a]_{v_{i}}$.

It's convenient to introduce some new elements in $\mathbf{U}_{h}$. Let

$$
v=e^{h / 2}, \quad \text { so } \quad v_{i}=v^{d_{i}}
$$

and for $1 \leq i \leq \ell$

$$
\begin{gathered}
K_{i}=v_{i}^{H_{i}}=e^{h d_{i} H_{i} / 2}, \\
E_{i}^{(n)}=E_{i}^{n} /[n]_{i}^{!}, \quad F_{i}^{(n)}=F_{i}^{n} /[n]_{i}^{!},
\end{gathered}
$$

where

$$
E_{i}=X_{i} K_{i}^{1 / 2}, \quad F_{i}=K_{i}^{-1 / 2} Y_{i} .
$$

Suppose $\mu=\sum_{i} a_{i} \alpha_{i} \in Y$. Denote $\prod_{i} K_{i}^{a_{i}}$ by $K_{\mu}$. 
One can put a Hopf algebra structure on $\mathbf{U}_{h}$ with coproduct $\Delta_{h}$, antipode $S_{h}$ and counit $\epsilon_{h}$ defined as follows.

$$
\begin{gathered}
\Delta_{h}\left(H_{i}\right)=H_{i} \otimes 1+1 \otimes H_{i}, \\
\Delta_{h}\left(X_{i}\right)=X_{i} \otimes K_{i}^{1 / 2}+K_{i}^{-1 / 2} \otimes X_{i}, \\
\Delta_{h}\left(Y_{i}\right)=Y_{i} \otimes K_{i}^{1 / 2}+K_{i}^{-1 / 2} \otimes Y_{i}, \\
\epsilon_{h}\left(H_{i}\right)=\epsilon_{h}\left(X_{i}\right)=\epsilon_{h}\left(Y_{i}\right)=0, \\
S_{h}\left(H_{i}\right)=-H_{i}, \quad S_{h}\left(X_{i}\right)=-K_{i} X_{i}, \quad S_{h}\left(Y_{i}\right)=-K_{i}^{-1} Y_{i} .
\end{gathered}
$$

Let $\mathbb{K}$ be the field of fractions of $\mathbb{C}[[h]]$ and $\mathbf{U}_{\mathbb{K}}=\mathbf{U}_{h} \otimes_{\mathbb{C}[[h]]} \mathbb{K}$. Let $\mathbf{U}_{v}$ be the $\mathbb{Q}(v)$-subalgebra of $\mathbf{U}_{\mathbb{K}}$ generated by $\left\{E_{i}, F_{i}, K_{i}\right\}_{1 \leq i \leq \ell}$. Let $\mathbf{U}_{\mathcal{A}}$ be the $\mathcal{A}$ subalgebra of $\mathbf{U}_{v}$ generated by $\left\{E_{i}^{(n)}, F_{i}^{(n)}, K_{i} \mid 1 \leq i \leq \ell, n \in \mathbb{N}\right\}$ where $\mathcal{A}=$ $\mathbb{Z}\left[v, v^{-1}\right]$. The Hopf algebra structure on $\mathbf{U}_{h}$ induces Hopf algebra structures on $\mathbf{U}_{\mathbb{K}}, \mathbf{U}_{v}$ and $\mathbf{U}_{\mathcal{A}}$. See Chapter 3 of $[\mathrm{L}$ for a proof of this fact.

Remark 2.1 Our $\mathbf{U}_{h}$ coincides with the one defined in $[\underline{\mathrm{K}}$ except that our $v_{i}$ is denoted $q_{i}$ there and because of this our $\mathbf{U}_{v}$ is denoted $U_{q}$ there. We use the same coproduct as in $[\mathrm{K}]$ which is opposite to the one used in $\left[\mathrm{L}\right.$. Our $\mathbf{U}_{v}$, $\mathbf{U}_{\mathcal{A}}$ and $K_{i}$ are denoted $\mathbf{U},{ }_{\mathcal{A}} \mathbf{U}$ and $\tilde{K}_{i}$ in $[\mathrm{L}$.

It is known that finite-dimensional simple modules of $\mathbf{U}_{\mathbb{K}}$ (resp. $\mathbf{U}_{v}$ ) are parameterized by the dominant weights of $\mathfrak{g}$, i.e. for any dominant weight $\lambda$ there is a unique finite-dimensional simple module $\tilde{V}_{\lambda}$ (resp. $V_{\lambda}$ ) of highest weight $\lambda$ and all finite-dimensional simple modules are of this form. Lusztig showed that $V_{\lambda}$ has a (canonical) basis $B(\lambda)$ such that the $\mathcal{A}$-module, denoted ${ }_{\mathcal{A}} V_{\lambda}$, generated by $B(\lambda)$ inherits a $\mathbf{U}_{\mathcal{A}}$-module structure from $V_{\lambda}$.

Let $\mathcal{V}_{\mathbb{K}}$ be the category of finite-dimensional $\mathbf{U}_{\mathbb{K}}$-modules of type I. The Hopf algebra structure on $\mathbf{U}_{\mathbb{K}}$ induces tensor product and duality in $\mathcal{V}_{\mathbb{K}}$. Any module $M$ from $\mathcal{V}_{\mathbb{K}}$ admits a weight space decomposition

$$
M=\bigoplus_{\lambda \in X} M^{\lambda}
$$

where $M^{\lambda}=\left\{x \in M \mid H_{i}(x)=\left(\alpha_{i} \mid \lambda\right) x\right\}$. Let $\mathcal{V}_{\mathcal{A}}$ be the category of finitely generated (as $\mathcal{A}$-modules) $\mathbf{U}_{\mathcal{A}}$-modules which have weight space decomposition as in Equation (11). Then ${ }_{\mathcal{A}} V_{\lambda}$ and its dual ${ }_{\mathcal{A}} V_{\lambda}{ }^{*}=\operatorname{Hom}_{\mathcal{A}}\left({ }_{\mathcal{A}} V_{\lambda}, \mathcal{A}\right)$ are objects of $\mathcal{V}_{\mathcal{A}}$ for $\lambda \in X_{+}$. Clearly $\mathcal{V}_{\mathcal{A}}$ is also a tensor category with duality. 
Let $\Theta$ and $\bar{\Theta}$ be the quasi- $R$-matrix and its inverse (Chapter 4 of $[\mathrm{L}]$ ). They are infinite sums

$$
\Theta=\sum_{s} a_{s} \otimes b_{s}, \quad \bar{\Theta}=\sum_{s} \bar{a}_{s} \otimes \bar{b}_{s}
$$

where $a_{s}, b_{s}, \bar{a}_{s}$ and $\bar{b}_{s}$ are in $\mathbf{U}_{v}$. So $\Theta$ and $\bar{\Theta}$ belong to some completion of $\mathbf{U}_{v} \otimes \mathbf{U}_{v}$. It turns out that they actually belong to some completion of $\mathbf{U}_{\mathcal{A}} \otimes \mathbf{U}_{\mathcal{A}}$, i.e. $a_{s}, b_{s}, \bar{a}_{s}$ and $\bar{b}_{s}$ are in $\mathbf{U}_{\mathcal{A}}$ (Corollary 24.1.6 of [L]). All the $a_{s}, b_{s}, \bar{a}_{s}$ and $\bar{b}_{s}$ act as 0 on any $M \in \mathcal{V}_{\mathbb{K}}$ except for finitely many of them. Therefore it makes sense to consider the map $\Theta$ (or $\bar{\Theta}): M \otimes N \rightarrow M \otimes N$ for any $M, N$ in $\mathcal{V}_{\mathbb{K}}$.

For $M, N$ in $\mathcal{V}_{\mathbb{K}}$, define $\Psi: M \otimes N \rightarrow M \otimes N$ by

$$
\Psi(x \otimes y)=v^{(\nu \mid \mu)} x \otimes y,
$$

if $x \in M^{\nu}$ and $y \in N^{\mu}$. Note that $(\nu \mid \mu)$ is not necessarily an integer 1 . Define the braiding operator $c_{M, N}$ as follows

$$
c_{M, N}=P \Psi \bar{\Theta}: M \otimes N \rightarrow N \otimes M
$$

where $P(x \otimes y)=y \otimes x$. It commutes with the $\mathbf{U}_{\mathbb{K}}$ action and hence is an operator from $\mathcal{V}_{\mathbb{K}}$.

Let $\Omega=\sum_{s} S_{h}\left(a_{s}\right) b_{s}$ where $a_{s}$ and $b_{s}$ are the ones in Equation (2). It is an element in some completion of $\mathbf{U}_{\mathcal{A}}$. For any $M \in \mathcal{V}_{\mathbb{K}}$ one can define $\Omega: M \rightarrow M$ because only finitely many terms in the summation act nontrivially. For such $M$ the twist operator 2 is

$$
\theta_{M}: M \rightarrow M, \quad \text { with } \quad \theta_{M}(x)=v^{(\nu+2 \rho \mid \nu)} \Omega(x),
$$

for $x \in M^{\nu}$. The operator $\theta_{M}$ is invertible and commutes with $\mathbf{U}_{\mathbb{K}}$ actions. If $M=\tilde{V}_{\lambda}, \lambda \in X_{+}$then $\theta_{M}=v^{(\lambda+2 \rho \mid \lambda)} \operatorname{Id}_{M}$.

The category $\mathcal{V}_{\mathbb{K}}$ is a ribbon category with braiding $c$ and twist $\theta$. But $\mathcal{V}_{\mathcal{A}}$ is not because of the fractional powers in Equations (3) and (5). We will define a ribbon subcategory of $\mathcal{V}_{\mathcal{A}}$ in Section 3.1

\subsection{TQFTs based on ribbon categories}

The objective of this section is to define TQFT of extended 3-cobordisms following Chapter IV of [T] with some modification. (See also Section 3.3 of [BK].)

\footnotetext{
${ }^{1}$ There exists an integer $\Delta$ for $\mathfrak{g}$ such that $(\nu \mid \mu)$ belongs to $\frac{1}{\Delta} \mathbb{Z}$ if $\nu, \mu \in X$ and belongs to $\mathbb{Z}$ if either $\nu$ or $\mu$ is in $Y$.

${ }^{2}$ The twist operator is called the quantum Casimir operator in Chapter 6 of $[\mathrm{L}$.
} 
Manifolds are always orientable and smooth. Maps between manifolds are always smooth. Non-zero (tangent or normal) vectors are equivalent up to scalar multiple. We fix a ribbon category $\mathcal{R}$ in the rest of this section. As mentioned in the introduction, a key feature of extended 3-cobordisms is that they contain colored ribbon graphs, which we define next.

\subsubsection{Ribbon graphs}

A band is a homeomorphic image of $[0,1]$ with a non-zero normal field. The images of 0 and 1 are called the bases of the band. An annulus is a homeomorphic image of $S^{1}$ with a non-zero normal field. A band or an annulus is oriented if it is equipped with a non-zero tangent field. A coupon is a homeomorphic image of $[0,1] \times[0,1]$. The images of $[0,1] \times 0$ and $[0,1] \times 1$ are called the bottom and the top of the coupon respectively. Coupons are always oriented.

Let $M$ be a 3 -manifold. A ribbon graph $\Omega$ in $M$ is a union of bands, annuli, and coupons embedded in $M$ such that:

(i) The bands and the annuli are oriented.

(ii) $\Omega$ meets $\partial M$ at some bases of the bands (called free ends of $\Omega$ ). The normal vectors of the bands at these free ends are tangent to $\partial M$.

(iii) Other bases of bands (called fixed ends of $\Omega$ ) lie on coupons' top or bottom with normal vectors in the direction of the positive side of the coupon.

(iv) Bands, annuli, and coupons are disjoint otherwise.

Let $\Omega$ be a ribbon graph. An $\mathcal{R}$-coloring of $\Omega$ is an assignment to each band and annulus of $\Omega$ an arbitrary object of $\mathcal{R}$, and to each coupon of $\Omega$ a morphism of $\mathcal{R}$ in the following way. Let $C$ be a coupon in $\Omega$ and we are looking at its positive side with the top above the bottom. Suppose the fixed ends on the bottom (resp. top) of $C$ have colors, counting from left to right, $V_{1}, \ldots, V_{m}$ (resp. $\left.W_{1}, \ldots, W_{n}\right)$. Let $\epsilon_{i}$ be $+($ resp. - ) if the $i$-th fixed end at the bottom of $C$ is oriented downward (resp. upward). Let $\delta_{j}$ be + (resp. - ) if the $j$-th fixed end at the top of $C$ is oriented downward (resp. upward). We assign $C$ a morphism

$$
f \in \operatorname{Hom}_{\mathcal{R}}\left(\bigotimes_{i=1}^{m} V_{i}^{\epsilon_{i}}, \bigotimes_{j=1}^{n} W_{j}^{\delta_{j}}\right)
$$

where we use the notation $V^{+}=V, V^{-}=V^{*}$ for any object $V$ of $\mathcal{R}$. A ribbon graph $\Omega$ together with an $\mathcal{R}$-coloring $\mu$ is called an $\mathcal{R}$-colored ribbon graph, denoted $\Omega(\mu)$. A ribbon graph is partially $\mathcal{R}$-colored if some bands and/or annuli and/or coupons are $\mathcal{R}$-colored. 


\subsubsection{Extended surfaces}

Another feature of extended 3-cobordisms is that their boundaries (or rather the boundaries of their underlying extended 3-manifolds, cf. 2.2.3) are extended surfaces. An $\mathcal{R}$-extended surface (or $e$-surface for short) is a closed oriented surface $\Gamma$ together with a finite set of $\mathcal{R}$-marks on it and a decomposable 3 Lagrangian subspace of $H_{1}(\Gamma, \mathbb{Q})$. An $\mathcal{R}$-mark on a closed surface $\Gamma$ is a point $p$ on $\Gamma$ associated with a triple $(t, V, \nu)$, where $t$ (direction of the mark) is a non-zero tangent vector at $p, V$ (color of the mark) is an arbitrary object from $\mathcal{R}$ and $\nu$ (sign of the mark) is + or - . If $\Gamma$ is an $e$-surface we denote by $-\Gamma$ the $e$-surface obtained from $\Gamma$ by reversing the orientation of $\Gamma$, keeping the Lagrangian subspace and, for every $\mathcal{R}$-mark on $\Gamma$, changing its sign while keeping its color and direction unchanged. The empty surface is considered as an $e$-surface which is not allowed to have any $\mathcal{R}$-mark on it.

An $e$-homeomorphism between two $e$-surfaces is a homeomorphism between the underlying surfaces that respects the extended structure, i.e. orientation, $\mathcal{R}$-marks and Lagrangian subspace.

\subsubsection{Extended 3-cobordisms}

Before defining extended 3-cobordisms we need another notion. An $\mathcal{R}$-extended 3-manifold is a triple $(M, \Omega, w)$ that consists of an oriented 3-manifold $M$ with $\mathcal{R}$-extended structure on $\partial M$, an integer $w$ (weight of $M$ ) and an $\mathcal{R}$-colored ribbon graph $\Omega$ (Section 2.2.1) sitting in it. The boundary of $M$ is an $e$-surface such that:

(i) The free ends of $\Omega$ meet $\partial M$ at the $\mathcal{R}$-marks only and each mark meets a free end of $\Omega$.

(ii) The colors (resp. normal vectors) of the bands of $\Omega$ and the colors (resp. directions) of the $\mathcal{R}$-marks agree if incident.

(iii) The sign of a mark is + (resp. - ) if the incident free end is directed inward (resp. outward).

(iv) The orientation on $\partial M$ is induced by that of $M$.

(v) It is required that $w(\emptyset)=0$.

\footnotetext{
${ }^{3}$ For a closed surface $\Gamma$ with connected components $\Gamma_{1}, \ldots, \Gamma_{m}$, a Lagrangian subspace of $H_{1}(\Gamma, \mathbb{Q})$ is decomposable if it is a direct sum of Lagrangian subspaces of $H_{1}\left(\Gamma_{i}, \mathbb{Q}\right), i=1, \ldots, m$.
} 
(There is no restriction on the Lagrangian subspace of $\partial M$.)

An $\mathcal{R}$-extended cobordism (or $e$-cobordism for short) is a triple $(M, \Gamma, \Lambda)$ where $M$ is an $\mathcal{R}$-extended 3-manifold and $\partial M=(-\Gamma) \sqcup \Lambda$ is an $e$-surface. An $e$ homeomorphism between two e-cobordisms is a homeomorphism between the underlying 3-manifolds that respects the extended structures on 3-manifolds and their boundaries.

Suppose $(M, \Gamma, \Lambda)$ and $\left(M^{\prime}, \Gamma^{\prime}, \Lambda^{\prime}\right)$ are two $e$-cobordisms and there is an $e$ homeomorphism $f: \Lambda \rightarrow \Gamma^{\prime}$. One can glue these two $e$-cobordisms along $f$ to get a new $e$-cobordism $\left(M \cup_{f} M^{\prime}, \Gamma, \Lambda^{\prime}\right)$. Here $M \cup_{f} M^{\prime}$ is an $\mathcal{R}$-extended 3 -manifold with an $\mathcal{R}$-colored ribbon graph (obtained by gluing gibbon graphs in $M$ and $M^{\prime}$ ) sitting inside and weight computed as in IV.9.1 of [T].

\subsubsection{TQFTs based on $\mathcal{R}$}

We finally can say what TQFTs really mean. Let $K$ be a commutative ring with unit and $\operatorname{Mod}(K)$ be the category of projective $K$-modules. A topological quantum field theory (TQFT) based on $\mathcal{R}$ with ground $\operatorname{ring} K$ is a pair $(\mathcal{T}, \tau)=$ $\left(\mathcal{T}_{\mathcal{R}}, \tau_{\mathcal{R}}\right)$. Here $\mathcal{T}$ is a modular functor $\mathbb{A}$ (based on $\mathcal{R}$ with ground ring $K$ ) from the category of $e$-surfaces and $e$-homeomorphisms to $\operatorname{Mod}(K)$ and $\tau$ assigns to every $e$-cobordism $(M, \Gamma, \Lambda)$ a $K$-homomorphism

$$
\tau(M)=\tau(M, \Gamma, \Lambda): \mathcal{T}(\Gamma) \rightarrow \mathcal{T}(\Lambda)
$$

that satisfies the following four axioms.

(Naturality) If $(M, \Gamma, \Lambda)$ and $\left(M^{\prime}, \Gamma^{\prime}, \Lambda^{\prime}\right)$ are two $e$-cobordisms and there is an $e$-homeomorphism $f: M \rightarrow M^{\prime}$ then $\tau\left(M^{\prime}\right) \circ \mathcal{T}\left(\left.f\right|_{\Gamma}\right)=\mathcal{T}\left(\left.f\right|_{\Lambda}\right) \circ \tau(M)$.

(Multiplicativity) $\tau\left(M \sqcup M^{\prime}\right)=\tau(M) \otimes_{K} \tau\left(M^{\prime}\right)$.

(Functoriality) If $(M, \Gamma, \Lambda)$ and $\left(M^{\prime}, \Gamma^{\prime}, \Lambda^{\prime}\right)$ are two $e$-cobordisms and there is an $e$-homeomorphism $f: \Lambda \rightarrow \Gamma^{\prime}$ then $\tau\left(M \cup_{f} M^{\prime}\right)=\tau\left(M^{\prime}\right) \circ \mathcal{T}(f) \circ \tau(M)$.

(Normalization) Suppose that $\Gamma$ is an $e$-surface. Then $\tau(\Gamma \times[0,1], \Gamma \times 0, \Gamma \times$ 1) $=\operatorname{Id}_{\mathcal{T}(\Gamma)}$

Here $(\Gamma \times[0,1], \Gamma \times 0, \Gamma \times 1)$ is the $e$-cobordism with weight 0 that is induced by the extended structure on $\Gamma$ in the obvious way. Let $(\mathcal{T}, \tau)$ be a TQFT based on $\mathcal{R}$ with ground ring $K$. For an $e$-cobordism $(M, \emptyset, \Gamma)$ one has $\tau(M)$ : $K \rightarrow \mathcal{T}(\Gamma)$. By slight abuse of notation, we denote $\tau(M)(1) \in \mathcal{T}(\Gamma)$ simply by

\footnotetext{
${ }^{4}$ A functor $\mathcal{T}$ is modular if $\mathcal{T}(\Gamma \sqcup \Lambda)$ is naturally identified with $\mathcal{T}(\Gamma) \otimes_{K} \mathcal{T}(\Lambda)$ and $\mathcal{T}(\emptyset)=K$, cf. III.1.2 of $[\mathrm{T}]$.
} 
$\tau(M)$. The TQFT is called non-degenerate if for any $e$-surface $\Gamma$, the module $\mathcal{T}(\Gamma)$ is generated over $K$ by the set

$$
\{\tau(M) \mid(M, \emptyset, \Gamma) \text { is an } e \text {-cobordism }\} .
$$

A cobordism with a closed underlying 3-manifold is called closed. The image of a closed cobordism $(M, \emptyset, \emptyset)$ under a TQFT is a linear map from $K$ to $K$. Therefore $\tau(M)=\tau(M)(1) \in K$. Let $D$ be a Dedekind domain contained in a ring $K$. Recall that A TQFT $(\mathcal{T}, \tau)$ with base ring $K$ is called almost (D-)integral if there exists some element $\mathcal{E} \in K$ such that $\mathcal{E} \tau(M)$ is in $D$ for any closed connected cobordism $(M, \emptyset, \emptyset)$.

\section{The main results}

In this section we construct an almost integral TQFT based on a ribbon category coming from $\mathbf{U}_{\mathcal{A}}$-modules.

Recall that $\mathfrak{g}$ is a simple complex Lie algebra. In what follows we fix an odd prime integer $r \geq d h^{\vee}$, which does not divide $\operatorname{det}\left(a_{i j}\right)$ in the notation of Section 2.1. We also fix a Cartan subalgebra $\mathfrak{h}$ of $\mathfrak{g}$ and a set of simple roots $\Pi_{\mathfrak{h}}=\left\{\alpha_{1}, \ldots, \alpha_{\ell}\right\}$ in its dual space $\mathfrak{h}^{*}$.

The fundamental alcove of level $k$ is defined as

$$
C_{k}=\left\{x \in \mathfrak{h}^{*} \mid\left(x \mid \alpha_{i}\right) \geq 0,\left(x \mid \alpha_{0}\right) \leq k, i=1, \ldots, \ell\right\}
$$

where $k=r-1-\left(\rho \mid \alpha_{0}\right)$ and $\alpha_{0}$ is the short highest root associated to the simple roots we choose. Note that $k \geq 0$ because $r \geq d h^{\vee}$. $\left(C_{k}\right.$ is equal to $\bar{C}_{k}$ in [L2.) The restriction that $r \nmid \operatorname{det}\left(a_{i j}\right)$ is to ensure the invertibility of the so-called $S$-matrix, cf. the paragraph before Example 3.2

\subsection{Four categories}

As mentioned in the introduction, we need to restrict the colors for the ribbon graphs in extended 3-cobordisms. To describe these colors we introduce the following 4 categories.

Let $\mathcal{V}_{1}=\mathcal{V}_{1}(\mathfrak{g}, r)$ be a category of $\mathbf{U}_{\mathcal{A}}$-modules over $\mathcal{A}$. Objects in $\mathcal{V}_{1}$ are direct summands of $V_{1} \star \cdots \star V_{n}$ where $V_{i}$ is ${ }_{\mathcal{A}} V_{\lambda_{i}}$ or its dual ${ }_{\mathcal{A}} V_{\lambda_{i}}{ }^{*}=\operatorname{Hom}_{\mathcal{A}}\left({ }_{\mathcal{A}} V_{\lambda_{i}}, \mathcal{A}\right)$ with $\lambda_{i} \in C_{k} \cap Y$ and $\star$ is either $\otimes_{\mathcal{A}}$ or $\oplus$. Morphisms in $\mathcal{V}_{1}$ are $\mathbf{U}_{\mathcal{A}}$-linear maps. So $\mathcal{V}_{1}$ is a full subcategory of $\mathcal{V}_{\mathcal{A}}$. Obviously $\mathcal{V}_{1}$ is closed under tensor product and duality. For any $\nu, \mu$ in the root lattice $Y,(\nu \mid \mu)$ is always an 
integer. The weights of an object from $\mathcal{V}_{1}$ are in $Y$, and so the braiding and the twist can be defined as in Equations (4) and (5) over the ring $\mathcal{A}$. Hence $\mathcal{V}_{1}$ is a ribbon category.

Let $\xi$ be a primitive $r$-th root of 1 . Consider $\mathbb{Z}[\xi]$ as an $\mathcal{A}$-algebra by sending $v$ to $\xi$. Let $\mathbf{U}_{\mathbb{Z}[\xi]}=\mathbf{U}_{\mathcal{A}} \otimes_{\mathcal{A}} \mathbb{Z}[\xi]$. Let $\mathcal{V}_{2}=\mathcal{V}_{2}(\mathfrak{g}, r)$ be a category of $\mathbf{U}_{\mathbb{Z}[\xi]^{-}}$ modules over $\mathbb{Z}[\xi]$. The objects in $\mathcal{V}_{2}$ are direct summands of $V \otimes_{\mathcal{A}} \mathbb{Z}[\xi]$ for $V$ in $\mathcal{V}_{1}$. Morphisms in $\mathcal{V}_{2}$ are $\mathbf{U}_{\mathbb{Z}[\xi]}$-morphisms. Obviously $\mathcal{V}_{2}$ is closed under tensor product and duality. The braiding and twist for $\mathcal{V}_{1}$ induce braiding and twist for $\mathcal{V}_{2}$. Hence, $\mathcal{V}_{2}$ is also a ribbon category.

Let $\mathbf{U}_{\mathbb{Q}(\xi)}=\mathbf{U}_{\mathbb{Z}[\xi]} \otimes_{\mathbb{Z}[\xi]} \mathbb{Q}(\xi)$ where $\mathbb{Q}(\xi)$ is the field of fractions of $\mathbb{Z}[\xi]$. Let $\mathcal{V}_{3}=\mathcal{V}_{3}(\mathfrak{g}, r)$ be a category of $\mathbf{U}_{\mathbb{Q}(\xi)}$-modules over $\mathbb{Q}(\xi)$. The objects in $\mathcal{V}_{3}$ are direct summands of $V \otimes_{\mathbb{Z}[\xi]} \mathbb{Q}(\xi)$ for $V$ in $\mathcal{V}_{2}$. Morphisms in $\mathcal{V}_{3}$ are $\mathbf{U}_{\mathbb{Q}(\xi)^{-}}$ morphisms. Similarly $\mathcal{V}_{3}$ is also a ribbon category.

Remark 3.1 The category $\mathcal{V}_{1}$ is different from the category of finite dimensional $\mathfrak{g}$-modules. For example, ${ }_{\mathcal{A}} V_{\lambda}^{*}$ is not isomorphic to ${ }_{\mathcal{A}} V_{-w_{0}(\lambda)}$ where $w_{0}$ is the longest element in $W$, cf. Section 3 of $\left[\mathrm{C}\right.$. The categories $\mathcal{V}_{2}$ and $\mathcal{V}_{3}$ are isomorphic as braided tensor categories in general. (We do not need this here. See [C] for a proof of this fact.) They are quite complicated. For example, they are not semisimple, i.e. they contain some objects that are reducible but indecomposable. To get a semisimple tensor category we need to quotient out some morphisms in $\mathcal{V}_{3}$.

We follow Section 3 of $[\underline{K}$ to define the last category. First note that objects in $\mathcal{V}_{3}$ are tilting modules. An object $V$ in $\mathcal{V}_{3}$ is called negligible if for every $f \in \operatorname{End}_{\mathcal{V}_{3}}(V)$ we have $\operatorname{tr}_{q}(f)=0$. Here $\operatorname{tr}_{q}(f)=\operatorname{tr}\left(K_{2 \rho} f\right)$ is the quantum trace of $f$. Then the tensor product of an arbitrary object with a negligible object is negligible. The dual of a negligible object is also negligible. These can be seen easily using graphical calculus, cf. I.2.7 of [T].

Let $V$ and $W \in \mathcal{V}_{3}$. A morphism $f: V \rightarrow W$ is negligible if $f=g g^{\prime}$ for some $g^{\prime}: V \rightarrow Z$ and $g: Z \rightarrow W$ such that $Z$ is negligible. Then for a negligible morphism $f: U \rightarrow U^{\prime}$ its transpose $f^{*}$ is negligible. For any morphism $g: V \rightarrow V^{\prime}, f \otimes g$ is negligible. If $U=V^{\prime}$ (resp. $V=U^{\prime}$ ) then $f g$ (resp. $g f$ ) is negligible. Hence it's possible to define the quotient category. (See also Section 3.3 of $[\mathrm{KT}$.) Let $\mathcal{N}=\mathcal{N}(\mathfrak{g}, r)$ be the clast 5 of negligible morphisms in $\mathcal{V}_{3}$. The quotient category $\mathcal{V}_{4}=\mathcal{V}_{4}(\mathfrak{g}, r)=\mathcal{V}_{3} / \mathcal{N}$ has the same objects as $\mathcal{V}_{3}$ and morphisms

$$
\operatorname{Hom}_{\mathcal{V}_{4}}(V, W)=\operatorname{Hom}_{\mathcal{V}_{3}}(V, W) / \text { negligible morphisms. }
$$

\footnotetext{
${ }^{5} \mathcal{N}$ is an ideal of $\mathcal{V}_{3}$ in the language of $[\mathrm{KT}]$.
} 
Any object $V$ in $\mathcal{V}_{1}$ (resp. $\mathcal{V}_{2}, \mathcal{V}_{3}$ ) gives rise to $\bar{V}=V \otimes \mathbb{Z}[\xi]$ (resp. $\hat{V}=$ $V \otimes \mathbb{Q}(\xi),[V]=V / \sim)$ in $\mathcal{V}_{2}$ (resp. $\mathcal{V}_{3}, \mathcal{V}_{4}$ ). Any morphism $f$ in $\mathcal{V}_{1}$ (resp. $\mathcal{V}_{2}$, $\mathcal{V}_{3}$ ) gives rise to $\bar{f}=f \otimes 1$ (resp. $\left.\hat{f}=f \otimes 1,[f]=f / \sim\right)$ in $\mathcal{V}_{2}\left(\right.$ resp. $\left.\mathcal{V}_{3}, \mathcal{V}_{4}\right)$. Here $\sim$ is the equivalence up to negligible morphisms. We denote ${ }_{\mathcal{A}} V_{\lambda},{ }_{\mathcal{A}} \bar{V}_{\lambda}$, ${ }_{\mathcal{A}} \hat{\bar{V}}_{\lambda}$ and $\left[{ }_{\mathcal{A}} \hat{\bar{V}}_{\lambda}\right]$ by ${ }_{1} V_{\lambda},{ }_{2} V_{\lambda},{ }_{3} V_{\lambda}$ and ${ }_{4} V_{\lambda}$ respectively.

Then $\mathcal{V}_{4}$ is a modular category that is dominated by simple objects ${ }_{4} V_{\lambda}, \lambda \in$ $C_{k} \cap Y$ (cf. Remark 3.10 of [K] 6 ). See [T] for the definition of modular categories. The invertibility of the so-called $S$ matrix in our case is proved in Theorem 3.3 of [L2], where $v$ is set to be a primitive $2 r$ th root of 1 . But this causes no problem. The $S$ matrix (before plugging in $\xi$ ) is a matrix over $\mathbb{Z}\left[v^{2}, v^{-2}\right]$ so in either case the entries of the $S$-matrix are substitutions of $v^{2}$ by a primitive $r$ th root of 1 . (Remember that $r$ is odd.)

Example 3.2 Let $\mathfrak{g}=s l_{2}$. The objects ${ }_{4} V_{\lambda}, \lambda=0, \alpha_{1}, \ldots, \frac{r-3}{2} \alpha_{1}$, are irreducible in $\mathcal{V}_{4}$ and every object in $\mathcal{V}_{4}$ is a direct sum of them. On the other hand, ${ }_{2} V_{\alpha_{1}} \otimes{ }_{2} V_{\frac{r-1}{2} \alpha_{1}}$ is not a direct sum of simple objects in $\mathcal{V}_{2}$.

\subsection{The TQFT}

In this section we define a TQFT, based on the ribbon category $\mathcal{V}_{2}$ with ground ring $\mathbb{Q}(\xi)$ (cf. Section 2.2.4), from the category of $\mathcal{V}_{2}$-extended cobordisms to $\mathcal{V}_{4}$. We modify the construction given in IV of $[\mathrm{T}]$. In the rest of this paper $e$-surfaces and $e$-cobordisms will mean $\mathcal{V}_{2}$-extended surfaces and $\mathcal{V}_{2}$-extended cobordisms.

\subsubsection{Parameterization of $e$-surfaces}

We also need to parametrize every e-surface by a standard surface, which by definition is either an empty surface or an $e$-surface that is the boundary of a standard handlebody (with induced extended structure). Here by a standard handlebody we mean a genus $g$ handlebody standardly embedded in $\mathbb{R}^{3}$ with a partially $\mathcal{V}_{2}$-colored oriented ribbon graph $R$ sitting inside as shown in Figure 1 . See Section 2.2.1 for the definition of ribbon graphs. The ribbon graph $R$ consists of a coupon (the narrow rectangle near the bottom), several vertical bands (with fixed ends on the coupon and free ends on the boundary of the standard handlebody) and $g$ half-circled bands (oriented to the left with bases

\footnotetext{
${ }^{6}$ The category $\mathcal{V}_{3}$, which is enough to produce a modular category, is a category of tilting modules contained in $\operatorname{Rep} U_{\xi}$ in $[\mathrm{K}]$. Our $d$ is denoted $m$ there and $\varkappa=r / 2 d$.
} 


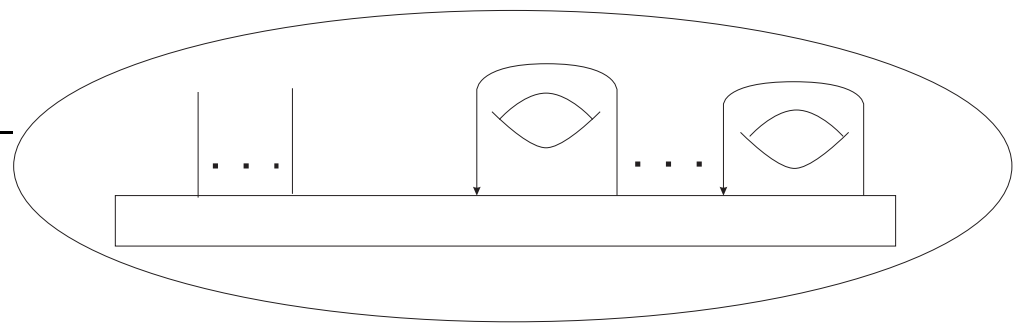

Figure 1: A standard handlebody

on the coupon). The vertical bands are oriented and colored by objects from $\mathcal{V}_{2}$. The coupon and the $g$ half-circled bands are not colored.

For any connected $e$-surface $\Gamma$ let

$$
\{p: \Sigma \rightarrow \Gamma \mid \Sigma \text { is a standard surface and } p \text { is an } e \text {-homeomorphism }\}
$$

be the set of all parameterizations of $\Gamma$ up to $e$-isotopy (in the obvious sense). For any $e$-surface $\Gamma$, this set is not empty (IV.6.4.2 of [T]). Note that $\Gamma$ may be parameterized by more than one standard surfaces because the $\mathcal{V}_{2}$-marks on $\Gamma$ are not ordered.

\subsubsection{The modular functor}

Let's define a modular functor $\mathcal{T}$. First, let $\mathcal{T}(\emptyset)=\mathbb{Q}(\xi)$.

Suppose $\Gamma$ is a genus $g$ e-surface with $m \mathcal{V}_{2}$-marks $\left(t_{i}, V_{i}, \epsilon_{i}\right), 1 \leq i \leq m$ (cf. Section 2.2.2). For each parameterization $p$ of $\Gamma$, put

$$
\mathcal{T}_{p}(\Gamma)=\bigoplus_{\lambda} \operatorname{Hom}_{\mathcal{V}_{4}}\left(\mathbb{Q}(\xi), \bigotimes_{i=1}^{m}\left[\hat{V}_{i}\right]^{\epsilon_{i}} \otimes \bigotimes_{i=1}^{g}\left({ }_{4} V_{\lambda_{i}} \otimes{ }_{4} V_{\lambda_{i}}{ }^{*}\right)\right)
$$

where $\lambda=\left(\lambda_{1}, \ldots, \lambda_{g}\right)$ ranges over $\left(C_{k} \cap Y\right)^{g}$. Recall from Section 3.1 that any object $V$ in $\mathcal{V}_{2}$ induces an object $[\hat{V}]$ in $\mathcal{V}_{4}$. Again $\left[\hat{V}_{i}\right]^{+}=\left[\hat{V}_{i}\right]$ and $\left[\hat{V}_{i}\right]^{-}=\left[\hat{V}_{i}\right]^{*}$.

Furthermore, for two parameterizations $p, p^{\prime}$ of $\Gamma$ there is an isomorphism $\varphi\left(p, p^{\prime}\right): \mathcal{T}_{p}(\Gamma) \rightarrow \mathcal{T}_{p^{\prime}}(\Gamma)$ of $\mathbb{Q}(\xi)$-vector spaces, defined as in IV.6.3 and IV.6.4.2 of [T]. Identify the vector spaces

$$
\left\{\mathcal{T}_{p}(\Gamma) \mid p \text { is a parameterization of } \Gamma\right\}
$$

along the isomorphisms $\left\{\varphi\left(p, p^{\prime}\right)\right\}_{\left(p, p^{\prime}\right)}$. The resulting vector spaces $\mathcal{T}(\Gamma)$ depends only on $\Gamma$. For any parameterization $p$ of $\Gamma, \mathcal{T}(\Gamma)$ is canonically isomorphic to $\mathcal{T}_{p}(\Gamma)$. Denote this isomorphism by $p_{\sharp}$. 
Let $\Gamma$ and $\Gamma^{\prime}$ be connected $e$-surfaces. For an $e$-homeomorphism $f: \Gamma \rightarrow \Gamma^{\prime}$ we define $\mathcal{T}(f): \mathcal{T}(\Gamma) \rightarrow \mathcal{T}\left(\Gamma^{\prime}\right)$ as follows. Pick any parameterization $p: \Sigma \rightarrow \Gamma$. Then $f p$ is a parameterization of $\Gamma^{\prime}$. Set $\mathcal{T}(f)=(f p)_{\sharp}\left(p_{\sharp}\right)^{-1}$ which does not depend on the chosen parameterization (cf. IV.6.3.1 of $[\mathrm{T}]$ ).

For non-connected $e$-surfaces we can do the above componentwisely and then form the tensor product. The above process defines a modular functor $\mathcal{T}$ from the category of $e$-surfaces and $e$-homeomorphisms to $\operatorname{Mod}(\mathbb{Q}(\xi))$ (cf. IV.6.3.3 of $[\mathrm{T}]$ ).

Remark 3.3 Unlike [T], which uses only a modular category in the construction, we start with the ribbon category $\mathcal{V}_{2}$ in the construction of the $e$-surfaces and $e$-cobordisms and use the modular category $\mathcal{V}_{4}$ later when we define the modular functor $\mathcal{T}$. This modification ensures that the TQFT constructed in the next subsection is almost integral. See also Remark 3.5 .

Remark 3.4 We quote several results from [T] in this and the next subsections. They are valid in our construction because the places where we use these results are the places where we are using the modular category $\mathcal{V}_{4}$.

\subsubsection{An almost integral TQFT}

Now we can describe the almost integral TQFT $(\mathcal{T}, \tau)$ as claimed at the beginning of Section 3. Let $\left(\mathcal{T}^{\mathcal{V}_{4}}, \tau^{\mathcal{V}_{4}}\right)$ be the TQFT derived from the modular category $\mathcal{V}_{4}$ in the way given in Section IV.9 of [T]. By definition, $\mathcal{T}^{\mathcal{V}_{4}}(\check{\Gamma})=\mathcal{T}(\Gamma)$ where $\check{\Gamma}$ denotes the $\mathcal{V}_{4}$-extended surface induced from $\Sigma$ by applying [^] (cf. Section 3.1) to the colors of the $\mathcal{V}_{2}$-marks on $\Gamma$. For a $\mathcal{V}_{2}$-extended cobordism $M$, we define $\tau(M)$ to be $\tau^{\mathcal{V}_{4}}(\check{M})$, where $\check{M}$ denotes the $\mathcal{V}_{4}$-extended cobordism induced from $M$ by applying [^] to the colors of the $\mathcal{V}_{2}$-extended ribbon graph in $M$ and the colors of the $\mathcal{V}_{2}$-marks on $\partial M$.

Lemma 3.1 The pair $(\mathcal{T}, \tau)$ is a non-degenerate TQFT, based on $\mathcal{V}_{2}$ with ground ring $\mathbb{Q}(\xi)$, from the category of $\mathcal{V}_{2}$-extended cobordisms to $\mathcal{V}_{4}$.

Proof The claim that $(\mathcal{T}, \tau)$ is a TQFT is almost immediate from the definition because we actually define the pair through a TQFT $\left(\mathcal{T}^{\mathcal{V}_{4}}, \tau^{\mathcal{V}_{4}}\right)$. We indicate why it's non-degenerate, i.e. $\mathcal{T}(\Gamma)$ is generated by $\tau(M)$ with $\partial M=\Gamma$. (Recall that we denote $\tau(M)(1)$ by $\tau(M)$.) It suffices to consider the case when $\Gamma$ is connected and $\Gamma=\Sigma$ for some standard surface $\Sigma$. 
Recall that $\Sigma$ bounds a standard handlebody $\mathcal{H}$ with a partially $\mathcal{V}_{2}$-colored ribbon graph $R$ sitting inside. Let $\mathcal{H}^{\prime}$ be the standard handlebody obtained from $\mathcal{H}$ by forgetting the $\mathcal{V}_{2}$-coloring on $R$. We denote the uncolored ribbon graph in $\mathcal{H}^{\prime}$ by $R^{\prime}$. Suppose the vertical bands in $R$ are colored by $V_{1}, \ldots, V_{m}$. By an admissible $\mathcal{V}_{4}$-coloring (or a-coloring for short) of $\mathcal{H}^{\prime}$ we mean a $\mathcal{V}_{4}$ coloring of $\mathcal{H}^{\prime}$ such that the $j$-th vertical band of $R^{\prime}$ is colored by $\left[\hat{V}_{j}\right]$ and the $i$-th half-circled band is colored by ${ }_{4} V_{\lambda_{i}}$ with $\lambda_{i} \in C_{k} \cap Y$. According to IV.2.1.3 of [T] the set $\left\{\tau^{\mathcal{V}_{4}}\left(\mathcal{H}^{\prime}(\mu)\right)\right\}_{\mu}$ generates $\mathcal{T}^{\mathcal{V}_{4}}(\check{\Sigma})=\mathcal{T}(\Sigma)$ where $\mu$ runs through all $a$-colorings of $\mathcal{H}^{\prime}$. We have to generate this set over $\mathbb{Q}(\xi)$ using $\mathcal{V}_{2}$-extended cobordisms.

Any morphism $f \in \mathcal{V}_{4}$ can be lifted (non-uniquely) to a morphism $f^{\prime} \in \mathcal{V}_{3}$, for which there exists $A \in \mathbb{Z}[\xi], A \neq 0$ such that $A f^{\prime}=\hat{f}^{\prime \prime}$ for some $f^{\prime \prime} \in \mathcal{V}_{2}$. Any $a$-coloring $\mu$ of $\mathcal{H}^{\prime}$ is uniquely determined by its color on the coupon, denoted $f_{\mu}$. We can find a $f_{\mu}^{\prime \prime} \in \mathcal{V}_{2}$ as above. Let $\mu^{\prime \prime}$ be the $\mathcal{V}_{2}$ coloring of $\mathcal{H}^{\prime}$ such that the coupon is colored by $f_{\mu}^{\prime \prime}$, the vertical bands are colored by $V_{j}$ and the half-circled bands are colored according to $f_{\mu}^{\prime \prime}$. Then $\mathcal{H}^{\prime}\left(\mu^{\prime \prime}\right)$ is a $\mathcal{V}_{2}$-colored cobordism. It's clear that $\tau\left(\mathcal{H}^{\prime}\left(\mu^{\prime \prime}\right)\right)=A \tau^{\mathcal{V}_{4}}\left(\mathcal{H}^{\prime}(\mu)\right)$ for some $A \in \mathbb{Z}[\xi], A \neq 0$. (The nonuniqueness of the lifts causes no trouble because the difference between two lifts of one morphism is negligible.)

Remark 3.5 We did not start with $\mathcal{V}_{4}$ because that would give too much freedom in coloring ribbon graphs and that in turn would destroy any integrality of the TQFT. Here is why. Recall that $\left(\mathcal{T}^{\mathcal{V}_{4}}, \tau^{\mathcal{V}_{4}}\right)$ is the TQFT based on $\mathcal{V}_{4}$ with ground ring $\mathbb{Q}(\xi)$ constructed as in $[\mathbb{T}$. We claim that it can not be almost integral. Suppose $D$ is a Dedekind domain in $\mathbb{Q}(\xi)$. Let $M$ be a closed connected $\mathcal{V}_{4}$-extended cobordism which contains a coupon colored by $f$. Let $M_{a}$ be the $\mathcal{V}_{4}$-extended cobordism obtained from $M$ by changing $f$ to af for some $a \in \mathbb{Q}(\xi)$. It's impossible to find a universal constant $\mathcal{E} \in \mathbb{Q}(\xi)$ such that $\mathcal{E} \tau\left(M_{a}\right) \in D$ for all $a \in \mathbb{Q}(\xi)$.

Recall that $\ell$ is the rank of $\mathfrak{g}$ and $w_{0}$ is the longest element in its Weyl group. Let $\zeta$ be a root of unity such that

$$
\operatorname{order}(\zeta)= \begin{cases}r & \text { if } \ell \text { is even, } r \text { is arbitrary and } \operatorname{sign}\left(w_{0}\right)=1, \\ r & \text { if } \ell \text { is odd and } \operatorname{sign}\left(w_{0}\right) r \equiv 1 \bmod 4, \\ 4 r & \text { otherwise }\end{cases}
$$

The following theorem is our main result which will be proved in Section 5 ,

Theorem 3.2 Let $r \geq d h^{\vee}$ be an odd prime that is not a factor of $\operatorname{det}\left(a_{i j}\right)$. Then the TQFT $(\mathcal{T}, \tau)$ is almost $\mathbb{Z}[\zeta]$-integral. 


\subsection{Calculating $\tau$}

Let $(M, \Omega, w)$ be a closed connected $\mathcal{V}_{2}$-extended 3-manifold. We recall how to calculate $\tau(M)$. Let $L$ be a framed link in $S^{3}$ such that $M$ is obtained from $S^{3}$ by surgery along $L$. Then there exists a $\mathcal{V}_{2}$-colored ribbon graph $\Omega^{\prime}$ in $S^{3}$ disjoint from $L$ such that $(M, \Omega)$ is obtained from $\left(S^{3}, \Omega^{\prime}\right)$ by surgery along $L$. By slight abuse of notation we will write $\Omega$ for $\Omega^{\prime}$.

Suppose that $L$ has $m$ components. Let $\mu=\left(\mu_{1}, \ldots, \mu_{m}\right) \in\left(C_{k} \cap Y\right)^{m}$. Denote by $L(\mu)$ the $\mathcal{V}_{2}$-colored framed link with the $i$-th component colored by ${ }_{2} V_{\mu_{i}}$. Let $U^{m}$ be the trivial link of $m$ components. Let $J=J^{\mathfrak{g}}$ be the quantum invariant of $\mathcal{V}_{2}$-colored ribbon graphs in $S^{3}$ defined in I.2.5 of [T]. Define

$$
F_{(L, \Omega)}=\sum_{\mu \in\left(C_{k} \cap Y\right)^{m}} J_{U^{m}(\mu)} J_{\Omega \sqcup L(\mu)} .
$$

We put $F_{ \pm}=F_{\left(U_{ \pm}, \emptyset\right)}$ for the trivial knot $U_{ \pm}$with \pm 1 framing. Let $\kappa$ and $\eta$ be one of the square roots of $F_{-} / F_{+}$and $F_{-} F_{+}$respectively such that $\kappa \eta=F_{-}$. (Note that $\eta$ and $F_{-}$are denoted $\mathcal{D}$ and $\Delta$ in $[\mathrm{T}$. Hence our $\kappa$ is $\Delta / \mathcal{D}$.) According to IV.9.2 and II.2.2 of [T]

$$
\begin{aligned}
\tau(M) & =\tau(M, \Omega, w)(1)=F_{-}^{\sigma} F_{(L, \Omega)} \eta^{-(\sigma+m+1)} \kappa^{w} \\
& =F_{(L, \Omega)} \eta^{-(m+1)} \kappa^{w+\sigma} \\
& =\frac{F_{(L, \Omega)}}{F_{-}^{\sigma_{-}+\beta_{1}} F_{+}^{\sigma_{+}}} \eta^{-1} \kappa^{\beta_{1}+w}
\end{aligned}
$$

where $\beta_{1}$ is the first Betti number of $M$ and $\sigma, \sigma_{+}$and $\sigma_{-}$are the signature, the number of positive eigenvalues and the number of negative eigenvalues of the linking matrix of $L$ respectively. Recall that $\sigma=\sigma_{+}-\sigma_{-}$and $m=\sigma_{+}+\sigma_{-}+\beta_{1}$.

\section{One application}

Again let $r \geq d h^{\vee}$ be an odd prime that is not a factor of $\operatorname{det}\left(a_{i j}\right)$ and let $\xi$ be a primitive $r$-th root of 1 . It is well known that for a closed connected oriented 3manifold $M$, the projective WRT-invariant $\tau_{M}^{P \mathfrak{g}}(\xi)$ is equal to $\eta \tau(M)$ multiplied by some algebraic integer for a universal complex number $\eta$, cf. Equation (8). Here $M$ is considered as a closed $\mathcal{V}_{2}$-extended cobordism with 0 weight and empty ribbon graph sitting inside. It is also known that $\tau_{M}^{P \mathfrak{g}}(\xi)$ is in $\mathbb{Z}[\xi]$.

One classical question in low dimensional topology is to determine whether a 3 -manifold is $p$-periodic. A manifold is $p$-periodic if it admits $\mathbb{Z} / p \mathbb{Z}$ action 
with fixed point set a circle. It is shown in GKP that if a homology sphere $M$ admits such an action then $\tau_{M}^{P s l_{2}}$ will satisfy some congruent relation. One can generalize it to all quantum invariants using Theorem 3.2

Corollary 4.1 CL Let $M$ be an $r$-periodic integral homology 3-sphere, and let $r, \xi$ be as above. Then

$$
\tau_{M}^{P \mathfrak{g}}(\xi) \equiv \xi^{s} \overline{\tau_{M}^{P \mathfrak{g}}(\xi)} \bmod r \text { in } \mathbb{Z}[\xi]
$$

for some integer $s$. Here 'bar' is the complex conjugation.

Remark 4.1 Let $P$ be the Poincare sphere ( -1 surgery on the left-hand trefoil), and let $B$ be the Brieskorn sphere ( -1 surgery on the right-hand trefoil). Corollary 4.1 is used in $\mathrm{CL}$ to show that $P$ has periodicity $2,3,5$ only and $B$ has periodicity $2,3,7$ only.

\section{Proofs}

\subsection{Proof of Theorem 3.2}

In this section we use Proposition 5.1, whose proof will be postponed till Section [5.2. to prove Theorem 3.2 Recall that $r$ is an odd prime $\geq d h^{\vee}$ which does not divide $\operatorname{det}\left(a_{i j}\right)$ and $\xi$ is a primitive $r$ th root of 1 . Let $\Omega \sqcup L$ be a ribbon graph in $S^{3}$ where $\Omega$ is a $\mathcal{V}_{2}$-colored ribbon graph (possibly with some $\mathcal{V}_{2}$-colored annulus components) and $L$ is an uncolored framed link of $m$ components.

Proposition 5.1 With the above notation, $F_{(L, \Omega)}$ is in $\mathbb{Z}[\xi]$ (cf. Equation [7)), and it is divisible by $(\xi-1)^{m(r \ell-\operatorname{dim} \mathfrak{g}) / 2}$ in $\mathbb{Z}[\xi]$.

This proposition will be proved in Section 5.2

Proof of Theorem 3.2 Let $(M, \Omega, w)$ be a closed connected $\mathcal{V}_{2}$-extended 3manifold. Suppose that $M$ is the result of surgery along a framed link $L \subset S^{3}$ of $m$ components. By Equation (8),

$$
\tau(M)=\frac{F_{(L, \Omega)}}{F_{-}^{\sigma_{-}+\beta_{1}} F_{+}^{\sigma_{+}}} \eta^{-1} \kappa^{\beta_{1}+w} .
$$

By Proposition 4.4 in $[\mathrm{L} 2], F_{ \pm} /(\xi-1)^{(r \ell-\operatorname{dim} \mathfrak{g}) / 2}$ is invertible in $\mathbb{Z}[\xi]$. Hence, using Proposition [5.1] $\frac{\vec{F}_{(L, \Omega)}}{F_{-}^{\sigma_{-}+\beta_{1} F_{+}^{\sigma_{+}}}}$is in $\mathbb{Z}[\xi]$. This implies that $\eta \tau(M) \in \mathbb{Z}[\xi, \kappa]$. The theorem now follows from the next lemma. 
Lemma 5.2 With the notations as above, $\kappa \in \mathbb{Z}[\zeta]$.

Sketch of proof First, one can follow the proof of Theorem 6.2 of $[\mathrm{K}$ to show that $\eta^{2}=F_{+} F_{-}=\operatorname{sign}\left(w_{0}\right) r^{\ell} / a^{2}$ for some $a \in \mathbb{Z}[\xi]$. Second, it's known that $\sqrt{\operatorname{sign}\left(w_{0}\right) r^{\ell}} \in \mathbb{Z}[\zeta]$. Hence $\eta$ belongs to $\mathbb{Q}(\zeta)$. Since $\eta \kappa=F_{-}$is in $\mathbb{Z}[\xi], \eta$ is in $\mathbb{Q}(\zeta)$ too. It remains to notice that $\kappa$ is always a root of unity (cf. again $[\mathrm{K}]$.

\subsection{Proof of Proposition 5.1}

The proof of Proposition 5.1 relies on the Kontsevich integral for framed tangles (cf. [LM] [KT]). The Kontsevich integral can be considered as a functor from the category of framed tangles to the category of chord diagrams. We define these two categories first.

\subsubsection{Categories of framed tangles and chord diagrams}

Up to the end of Section [5.2.3, we follow [KT] closely so that the reader can easily find some missing details there. For a different approach see [LM].

Let $\mathbb{T}$ be the category of framed tangles. The objects are words in +'s and -'s and the morphisms are framed tangles (up to the equivalence classes) in $\mathbb{R}^{2} \times[0,1]$. The category $\mathbb{T}$ is a strict ribbon category (cf. Section 2.1 of $[\mathrm{KT}$, where $\mathbb{T}$ is denoted $\mathcal{T}$ ).

For any pair of non-negative integers $(a, b)$, an $(a, b)$-curve $C$ is a compact oriented smooth curve 7 such that $\partial C$ is divided into two totally ordered sets, source $s(C)$ and target $t(C)$ of cardinalities $a$ and $b$ respectively. An $(a, b)$ chord diagram is an $(a, b)$-curve $C$ together with finitely many pairs of unordered points (called chord-ends) on $C$. Such a pair is usually indicated by a dashed line (called a chord on $C$ ) connecting the pair. Let $\mathbb{A}$ be the category of chord diagrams. An object (resp. a morphism) in $\mathbb{A}$ is a word in +'s and -'s (resp. a $\mathbb{C}$-linear combination of chord diagrams (up to the equivalence classes) with the same target and source). Then $\mathbb{A}$ is a strict infinitesimal symmetric category. In Section 2.3 of $[\mathrm{KT}, \mathbb{A}$ is denoted $\mathcal{A}(\mathbb{C})$. Note that chord diagrams in [KT] are equipped with residues, an extra structure being omitted here for it is not essential for our purpose.

\footnotetext{
${ }^{7}$ Smooth curves are smooth 1-manifolds. They do not have to be embedded in 3-manifolds nor have associated normal vectors.
} 


\subsubsection{The Kontsevich integral for framed tangles}

For any strict infinitesimal symmetric category one can obtain a braided tensor category using formal integral, cf. the proof of Theorem 4.7 of [KT]. We recall it when the strict infinitesimal symmetric category is $\mathbb{A}$.

Let $h$ be a formal parameter. The formal integral $\mathbb{A}[[h]]$ of $\mathbb{A}$ is a category whose objects are the same as those of $\mathbb{A}$ and whose morphisms from $s$ to $s^{\prime}$ are formal series $\sum_{i=0}^{\infty} f_{i} h^{i}$ with $f_{i} \in \operatorname{Hom}_{\mathbb{A}}\left(s, s^{\prime}\right)$. There exists a braided tensor category structure on $\mathbb{A}[[h]]$. This category is not a strict braided tensor category.

Let $\mathbb{A}^{\prime}=\mathbb{A}[[h]]^{\text {str }}$ be the strictified category of $\mathbb{A}[[h]]$. See XI.5 of $[\mathbf{K}]$ for the definition of strictification. Then $\mathbb{A}^{\prime}$ is a strict braided tensor category whose objects are finite sequences of objects of $\mathbb{A}$, including the empty sequence. For such a sequence $s=\left(s_{1}, \ldots, s_{k}\right)$, let $P(s)=\otimes_{i} s_{i}$ if $k>0$, where tensor product is taken in the order of index $i$, and $P(s)=\mathbb{C}$ if $k=0$. For any two objects $s$ and $s^{\prime}$ in $\mathbb{A}^{\prime}$ let

$$
\operatorname{Hom}_{\mathbb{A}^{\prime}}\left(s, s^{\prime}\right)=\operatorname{Hom}_{\mathbb{A}[[h]]}\left(P(s), P\left(s^{\prime}\right)\right) .
$$

Lemma 5.3 $\left[\mathrm{KT}\right.$ Section 6 There is a functor $Z: \mathbb{T} \rightarrow \mathbb{A}^{\prime}$ respecting braided tensor category structure.

The functor $Z$ is called the Kontsevich integral for framed tangles. For any framed tangle $T$,

$$
Z(T)=\sum_{i=0}^{\infty} Z_{i}(T) h^{i}
$$

where $Z_{i}(T)$ is a linear combination of chord diagrams with $i$ chords, whose underlying 1-manifolds are $T$ forgetting framing. We call $Z_{i}(T)$ the degree $i$ part of $Z(T)$.

\subsubsection{A weight system}

To calculate the quantum invariant $J^{\mathfrak{g}}$ from the Kontsevich integral we need to define a weight system. We are only interested in the string tangle case. A string tangle is a tangle whose $i$-th free upper end is connected to the $i$-th free lower end for all $i$. A string tangle is allowed to have closed components. A string chord diagram is defined similarly.

Let $V$ be a finite dimensional $\mathfrak{g}$-module. Let $C: \epsilon_{1} \cdots \epsilon_{m} \rightarrow \epsilon_{1} \cdots \epsilon_{m}$ be a string chord diagram. It is a morphism in $\mathbb{A}$. A state $s_{0}$ of $C$ is an assignment 
to each unordered pair of points (chord) an element $x_{i}, i \in\{1, \ldots, \operatorname{dim} \mathfrak{g}\}$ where $\left\{x_{i}\right\}$ is an orthonormal basis of $\mathfrak{g}$ with respect to the Killing form. For each component of the underlying 1-manifold of $C$ we get an element in the universal enveloping algebra $\mathbf{U}(\mathfrak{g})$ obtained by multiplying $x_{i}$ 's on this component in the order of the orientation of this component. Thus we get $n$ elements $y_{j}$ in $\mathbf{U}(\mathfrak{g})$ where $n$ is the number of components of $C$. Tensoring up these $n$ elements one gets an element in $\mathbf{U}(\mathfrak{g})^{\otimes n}$. If the $i$-th component of $C$ is an annulus then we take the trace $\operatorname{tr}_{V}\left(y_{i}\right)$. Having done so for all annulus components we end up with $w\left(s_{0}\right) \in \mathbf{U}(\mathfrak{g})^{\otimes m}$. The weight of $C$ is

$$
\mathfrak{w}_{\mathfrak{g}}(C)=\sum_{s_{0}} w\left(s_{0}\right): \bigotimes_{i=1}^{m} V^{\epsilon_{i}} \rightarrow \bigotimes_{i=1}^{m} V^{\epsilon_{i}}
$$

summed over all possible states of $C$, where $V^{+}=V$ and $V^{-}=V^{*}$. Note that although $y_{i}$ is not well-defined for an annulus component, the weight is welldefined because we take the trace. We extend the weight to linear combination of chord diagrams by linearity.

We will use $Z$ and $\mathfrak{w}_{\mathfrak{g}}$ to compute $J$ in Equation (15). See also Remark 5.1.

\subsubsection{A lemma on $J$}

The symmetric algebra $\mathfrak{S}\left(\Pi_{\mathfrak{h}}\right)$ in the simple roots $\Pi_{\mathfrak{h}}$ can be considered as polynomials on the root lattice as follows. For any monomial $p=\alpha_{i_{1}} \cdots \alpha_{i_{n}}$ in $\mathfrak{S}\left(\Pi_{\mathfrak{h}}\right)$ and any $\mu \in Y$, let

$$
p(\mu)=\left(\alpha_{i_{1}} \mid \mu\right) \cdots\left(\alpha_{i_{n}} \mid \mu\right)
$$

(cf. [L1]). The degree of $p$ is $n$.

Recall from Section 3.3 that $J=J^{\mathfrak{g}}$ is the quantum invariant of $\mathcal{V}_{2}$-colored ribbon graphs in $S^{3}$ defined in I.2.5 of [T]. Suppose that $\Omega$ is a $\mathcal{V}_{2}$-colored ribbon graph is $S^{3}$. Let $L$ be an $m$-component link in $S^{3}$ disjoint from $\Omega$. Let $\mu=\left(\mu_{1}, \ldots, \mu_{m}\right)$ with $\mu_{i} \in Y \cap C_{k}$. Denote by $L(\mu)$ the $\mathcal{V}_{2}$-colored framed link with the $i$-th component colored by ${ }_{2} V_{\mu_{i}}$. Therefore $\Omega \sqcup L(\mu)$ is a $\mathcal{V}_{2}$-extended ribbon graph. The following lemma deals with the dependence of $J_{\Omega \sqcup L(\mu)}$ on $\mu_{1}, \ldots, \mu_{m}$. Again, we assume that $r$ is an odd prime $\geq d h^{\vee}$ which does not divide $\operatorname{det}\left(a_{i j}\right)$ and $\xi$ is a primitive $r$-th root of 1 .

Lemma 5.4 In the above notation,

$$
J_{\Omega \sqcup L(\mu)}=\left.\sum_{i=0}^{m(r \ell-\operatorname{dim} \mathfrak{g}) / 2-1} f_{i}\left(\mu_{1}, \ldots, \mu_{m}\right)(v-1)^{i}\right|_{v=\xi}+R
$$




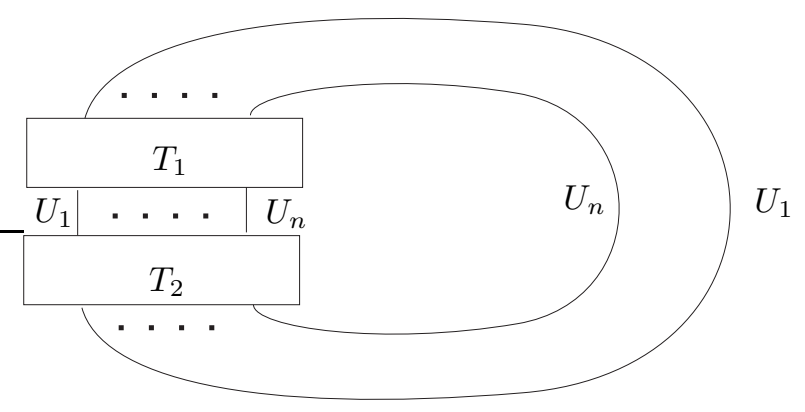

Figure 2: A tangle presentation for $\Omega \sqcup L$

where $f_{i}$ is a polynomial in $\mu_{1}, \cdots, \mu_{m}$ of total degree not exceeding $2 i+m s$ for all $i$ and $R \in \mathbb{Z}[\xi]$ is divisible by $(\xi-1)^{m(r \ell-\operatorname{dim} \mathfrak{g}) / 2}$ in $\mathbb{Z}[\xi]$. Here $s$ is the number of positive roots of $\mathfrak{g}$. Furthermore, for any $i$, the polynomial $f_{i}$ takes integer values for $\mu_{1}, \ldots, \mu_{m} \in C_{k} \cap Y$.

Proof It's obvious that $\Omega \sqcup L$ can be presented as a diagram shown in Figure 2 . where $T_{2}$ is the diagram of a string tangle and $T_{1}$ is the diagram of a ribbon graph such that all coupons of $\Omega$ are inside $T_{1}$ and the link $L$ is inside $T_{2}$. To see this we first draw an arbitrary diagram presenting $\Omega \sqcup L$ inside a disk $D_{1}$. Then we pull $L$ into a disk $D_{2}$ disjoint from $D_{1}$ keeping all coupons inside $D_{1}$. After that the strings connecting $D_{1}$ and $D_{2}$, which are disjoint from $L$, can be arranged into the diagram shown in Figure 2. Hence

$$
J_{\Omega \sqcup L(\mu)}=\operatorname{tr}_{q}\left(J_{T_{1}} \circ J_{T_{2}(\mu)}\right)=\operatorname{tr}\left(J_{T_{1}} \circ J_{T_{2}(\mu)} \circ K_{2 \rho}\right) .
$$

Suppose that the strings connecting $T_{1}$ and $T_{2}$ have colors $U_{1}, \ldots, U_{n}$. Then both $J_{T_{1}}$ and $J_{T_{2}(\mu)}$ are endomorphisms of $U=\otimes_{i} U_{i}^{\epsilon_{i}}$ where $\epsilon_{i}= \pm$ is determined by the orientation of the $i$-th string, cf. Section 2.2.1]

Recall from Section 3.1 that for any object $V$ in $\mathcal{V}_{2}$ there exists a unique object $V^{\prime}$ in $\mathcal{V}_{1}$ such that $V=\bar{V}^{\prime}$. Pick a basis $B^{\prime}$ for $U^{\prime}$ which induces a basis $B$ for $U$. Then $J_{T_{1}}$ is a matrix over $\mathbb{Z}[\xi]$ with respect to $B$. Let $\hat{J}_{T_{1}}$ be the matrix over $\mathcal{A}$ obtained from $J_{T_{1}}$ by substituting $\xi$ by $v$. Note that $\hat{J}_{T_{1}}$ is not well-defined. For example $\xi^{r}$ can be substituted by either $v^{r}$ or 1 . But one certainly has $\left.\hat{J}_{T_{1}}\right|_{v=\xi}=J_{T_{1}}$.

Let $T_{2}\left(\mu^{\prime}\right)$ be a $\mathcal{V}_{1}$-colored framed string tangle obtained from $T_{2}(\mu)$ by changing color on each of its component, including the bands, from $V$ to $V^{\prime}$. Note that $T_{2}$ contains no coupons. Let $J^{\prime}$ be the invariant for $\mathcal{V}_{1}$-colored ribbon graphs constructed in Theorem I.2.5 of [T]. Then $J_{T_{2}\left(\mu^{\prime}\right)}^{\prime}: U^{\prime} \rightarrow U^{\prime}$ is a morphism in $\mathcal{V}_{1}$ which is presented as a matrix over $\mathcal{A}$ with respect to $B^{\prime}$. Since 
the ribbon structures on $\mathcal{V}_{1}$ and $\mathcal{V}_{2}$ are induced by the same quasi- $R$-matrix $\Theta$ (cf. Equations (2[5) ), $\left.J_{T_{2}\left(\mu^{\prime}\right)}^{\prime}\right|_{v=\xi}$ is the matrix over $\mathbb{Z}[\xi]$ presenting $J_{T_{2}(\mu)}$ with respect to $B$. We have

$$
J_{\Omega \sqcup L(\mu)}=\left.\operatorname{tr}\left(\hat{J}_{T_{1}} J_{T_{2}\left(\mu^{\prime}\right)}^{\prime} K_{2 \rho}\right)\right|_{v=\xi},
$$

where $\hat{J}_{T_{1}} J_{T_{2}\left(\mu^{\prime}\right)}^{\prime} K_{2 \rho}$ is considered as a product of matrices. By Lemma [5.5] below we have

$$
\left.\operatorname{tr}\left(\hat{J}_{T_{1}} J_{T_{2}\left(\mu^{\prime}\right)}^{\prime} K_{2 \rho}\right)\right|_{v=e^{h / 2}}=\sum_{i=0}^{\infty} p_{i}\left(\mu_{1}, \ldots, \mu_{m}\right) h^{i}
$$

where $p_{i}$ is a polynomial in root lattice of degree at most $2 i+m s$ for all $i$. (Note that $K_{2 \rho}$ and $\hat{J}_{T_{1}}$ are independent of $\mu$.) Substituting $v$ back into Equation (13), i.e. letting

$$
h=2 \ln (v)=2 \sum_{i=1}^{\infty}(-1)^{i-1}(v-1)^{i} / i,
$$

we have

$$
\begin{aligned}
\operatorname{tr}\left(\hat{J}_{T_{1}} J_{T_{2}\left(\mu^{\prime}\right)}^{\prime} K_{2 \rho}\right) & =\sum_{i=0}^{\infty} f_{i}\left(\mu_{1}, \ldots, \mu_{m}\right)(v-1)^{i} \\
& =\sum_{i=0}^{m(r \ell-\operatorname{dim} \mathfrak{g}) / 2-1} f_{i}\left(\mu_{1}, \ldots, \mu_{m}\right)(v-1)^{i}+R_{v}
\end{aligned}
$$

with $R_{v}$ divisible by $(v-1)^{m(r \ell-\operatorname{dim} \mathfrak{g}) / 2}$ in $\mathcal{A}$. The element $R_{v}$ is a priori in $\mathbb{C}[[v]]$. It belongs to $\mathcal{A}$ follows from the fact that $\hat{J}_{T_{1}} J_{T_{2}\left(\mu^{\prime}\right)}^{\prime} K_{2 \rho}$ can be presented as a matrix over $\mathcal{A}$ with respect to basis $B^{\prime}$ if $\mu_{i} \in Y_{+}$. For the same reason we see that $f_{i}$ takes integer values if $\mu_{i} \in Y_{+}$. The coefficients $f_{i}$ are polynomials in root lattice of degree at most $2 i+m s$. Substituting $v$ by $\xi$ we get Equation (11).

Before we state Lemma [5.5] let's recall a fact first.

\subsubsection{A fact about $\mathrm{U}(\mathfrak{g})$}

Any element $x$ in $\mathbf{U}(\mathfrak{g})$ can be written uniquely as a sum $x=z+u$ for some $z$ in $\operatorname{center}(\mathbf{U}(\mathfrak{g}))$ and $u$ in $[\mathbf{U}(\mathfrak{g}), \mathbf{U}(\mathfrak{g})]$ such that $\operatorname{deg}(z) \leq \operatorname{deg}(x)$ (cf. Page 
105 Exercise 19 of $[\mathrm{B}]$ or Section 2.3.3 of $\left[\mathrm{W}^{8}\right)$. Here deg is the PBW-degree. Let $V_{\lambda}$ be the simple $\mathfrak{g}$-module with highest weight $\lambda \in Y_{+}$. Then $z$ acts on $V_{\lambda}$ as a scalar $z(\lambda) \in \mathbb{C}$. By the Harish-Chandra theory $z(\lambda)$ is a polynomial function on root lattice with degree no greater than $\operatorname{deg}(z)$. Then by the Weyl formula the trace $\operatorname{tr}_{V_{\lambda}}(z)=\operatorname{dim}\left(V_{\lambda}\right) z(\lambda)$ is a polynomial on root lattice with degree at most $\operatorname{deg}(z)+s$. Since $u$ has trace 0 it follows that $\operatorname{tr}_{V_{\lambda}}(x)$ is also a polynomial in root lattice which has degree at most $\operatorname{deg}(x)+s$.

\subsubsection{A lemma on $J^{\prime}$}

Recall that $J^{\prime}$ is the invariant for $\mathcal{V}_{1}$-colored ribbon graphs constructed in Theorem I.2.5 of [T]. The following lemma is a generalization of Lemma 4.6 of L2].

Lemma 5.5 For $\nu=\left(\nu_{1}, \ldots, \nu_{m}\right)$, let $T(\nu)$ be a $\mathcal{V}_{1}$-colored framed string tangle whose closed components are colored by ${ }_{1} V_{\nu_{1}}, \ldots,{ }_{1} V_{\nu_{m}}$. Then, $J_{T(\nu)}^{\prime}$ can be presented by a matrix $\left(c_{i j}\right)$ such that

$$
\left.c_{i j}\right|_{v=e^{h / 2}}=\sum_{k=0}^{\infty} p_{k}\left(\mu_{1}, \ldots, \mu_{m}\right) h^{k}
$$

where $p_{k}$ is a polynomial in $\mu_{1}, \ldots, \mu_{m}$ of total degree not exceeding $2 i+m s$.

Let $\mathcal{V}_{\mathfrak{g}}$ be the category of finite dimensional $\mathfrak{g}$-modules. It is well known that for each object $V$ in $\mathcal{V}_{1}$ there exists a unique object $V_{\mathfrak{g}}$ in $\mathcal{V}_{\mathfrak{g}}$ with the same weight space as $V$.

Proof Let $T(\tilde{\nu})$ be the $\mathcal{V}_{\mathfrak{g}}$-colored framed string tangle obtained from $T(\nu)$ by changing all colors from $V$ to $V_{\mathfrak{g}}$. Then one can calculate $J_{T(\nu)}^{\prime}$ from the Kontsevich integral (Equation (92))

$$
Z(T(\tilde{\nu}))=\sum_{i=0}^{\infty} Z_{i}(T(\tilde{\nu})) h^{i}
$$

as follows. Suppose that $J_{T(\nu)}^{\prime}$ is an endomorphism of $V \in \mathcal{V}_{1}$. Then it follows that $\mathfrak{w}_{\mathfrak{g}}\left(Z_{i}(T(\tilde{\nu}))\right)$ is an endomorphism of $V_{\mathfrak{g}} \in \mathcal{V}_{\mathfrak{g}}$, cf. Equation (10). For any basis of $V$ and of $V_{\mathfrak{g}}$ there exists an invertible matrix $A$ over $\mathbb{C}[[h]]$, independent of $\nu$, such that (as matrices over $\mathbb{C}[[h]]$ ) we have

$$
\left.J_{T(\nu)}^{\prime}\right|_{v=e^{h / 2}}=A\left(\sum_{i=0}^{\infty}(-1)^{i} \mathfrak{w}_{\mathfrak{g}}\left(Z_{i}(T(\tilde{\nu}))\right) h^{i}\right) A^{-1} .
$$

\footnotetext{
${ }^{8}$ We thank the referee for pointing out this reference to us.
} 
(See Remark 5.1 below.)

Hence each entry of $\left.J_{T(\nu)}^{\prime}\right|_{v=e^{h / 2}}$ is of the form

$$
\sum_{k=0}^{\infty} p_{k}\left(\nu_{1}, \ldots, \nu_{m}\right) h^{k}
$$

where $p_{k}$ is a polynomial in $\nu_{1}, \ldots, \nu_{m}$ of total degree not exceeding $2 i+m s$ (each chord has two chord-ends). Now Equation (14) follows from this and (15).

Remark 5.1 Equation (15) is proved in Theorem 7.2 of [KT]. Strictly speaking, one needs to generalize the Kontsevich integral $Z$ in Section 5.2 .2 (resp. weight system $\mathfrak{w}_{\mathfrak{g}}$ in Section 5.2.3) to the case when components of tangles (resp. chord diagrams) are colored by (not necessarily identical) objects in $\mathcal{V}_{\mathfrak{g}}$. But this generalization is quite straightforward.

\subsubsection{Proof of Proposition 5.1}

Since $J$ is defined over $\mathcal{V}_{2}, F_{(L, \Omega)} \in \mathbb{Z}[\xi]$. By Lemma 5.4

$$
J_{U^{m}(\mu)} J_{\Omega \sqcup L(\mu)}=\sum_{i=0}^{m(r \ell-\operatorname{dim} \mathfrak{g}) / 2-1} f_{i}^{\prime}\left(\mu_{1}, \ldots, \mu_{m}\right)(\xi-1)^{i}+R^{\prime}
$$

where $R^{\prime} \in \mathbb{Z}[\xi]$ is divisible by $(\xi-1)^{m(r \ell-\operatorname{dim} \mathfrak{g}) / 2}$ and $f_{i}^{\prime}$ 's are polynomials in root lattice of degree at most $2 i+2 m s$ taking integer values for $\mu_{i} \in Y_{+} \cap C_{k}$. Since

$$
F_{(L, \Omega)}=\sum_{\mu \in\left(Y_{+} \cap C_{k}\right)^{m}} J_{U^{m}(\mu)} J_{\Omega \sqcup L(\mu)}
$$

we can apply Lemma 4.7 in $\left[{ }^{9}\right.$ to show that $F_{(L, \Omega)}$ is divisible by $(\xi-$ $1)^{m(r \ell-\operatorname{dim} \mathfrak{g}) / 2}$ in $\mathbb{Z}[\xi]$. This ends the proof of Proposition 5.1 .

\section{References}

[BK] B Bakalov, A Kirillov, Jr, Lectures on tensor categories and modular functors, University Lecture Series 21, American Mathematical Society, Providence, RI (2001) MathReview

\footnotetext{
${ }^{9}$ The proof of this lemma refers to Corollary 4.14 of [L1], which should be Corollary 4.11 instead.
} 
[BHMV] C Blanchet, N Habegger, G Masbaum, P Vogel, Topological quantum field theories derived from the Kauffman bracket, Topology 34 (1995) 883-927 MathReview

[B] N Bourbaki, Lie groups and Lie algebras. Chapters 1-3, Elements of Mathematics (Berlin), Springer-Verlag, Berlin (1989) MathReview

[C] Qi Chen, On certain integral tensor categories and integral TQFTs, arXiv:math.QA/0408356

[CL] Q Chen, T TQ Le, Quantum invariants of periodic links and periodic 3manifolds, Fund. Math. 184 (2004) 55-71 MathReview

[GKP] P M Gilmer, J Kania-Bartoszynska, J H Przytycki, 3-manifold invariants and periodicity of homology spheres, Alg. Geom. Topol. 2 (2002) 825-842 MathReview

[G] P M Gilmer, Integrality for TQFTs, Duke Math. J. 125 (2004) 389-413 MathReview

[K] C Kassel, Quantum groups, Graduate Texts in Mathematics 155, SpringerVerlag, New York (1995) MathReview

[KT] C Kassel, V Turaev, Chord diagram invariants of tangles and graphs, Duke Math. J. 92 (1998) 497-552 MathReview

$[\mathrm{K}] \quad$ A A Kirillov, Jr, On an inner product in modular tensor categories, J. Amer. Math. Soc. 9 (1996) 1135-1169 MathReview

[L1] T T Q Le, On perturbative PSU $(n)$ invariants of rational homology 3-spheres, Topology 39 (2000) 813-849 MathReview

[L2] T T Q Le, Quantum invariants of 3-manifolds: integrality, splitting, and perturbative expansion, Topology Appl. 127 (2003) 125-152 MathReview

[LM] TQT Le, J Murakami, The universal Vassiliev-Kontsevich invariant for framed oriented links, Compositio Math. 102 (1996) 41-64 MathReview

[L] G Lusztig, Introduction to quantum groups, Progress in Mathematics 110, Birkhäuser Boston Inc. Boston, MA (1993) MathReview

[RT] N Reshetikhin, V G Turaev, Invariants of 3-manifolds via link polynomials and quantum groups, Invent. Math. 103 (1991) 547-597 MathReview

[T] V G Turaev, Quantum invariants of knots and 3-manifolds, de Gruyter Studies in Mathematics 18, Walter de Gruyter \& Co. Berlin (1994) MathReview

[W] G Warner, Harmonic analysis on semi-simple Lie groups, I, Grundlehren series 188, Springer-Verlag (1972) MathReview

Department of Mathematics, The Ohio State University

Columbus, OH 43210-1174, USA

and

School of Mathematics, Georgia Institute of Technology

Atlanta, GA 30332-0160, USA

Email: qichen@math.ohio-state.edu, letu@math.gatech.edu

Received: 26 September 2003 Revised: 16 September 2005 